\title{
QoS-Aware Multilayer UAV Deployment to Provide VoWiFi Service over 5G Networks
}

\author{
Vicente Mayor $(\mathbb{D}$, Rafael Estepa $(\mathbb{D}$, and Antonio Estepa \\ Department of Telematics Engineering, Escuela Superior de Ingenieros, Universidad de Sevilla, C/Camino de los Descubrimientos s/ \\ n, 41092 Sevilla, Spain \\ Correspondence should be addressed to Vicente Mayor; vmayor@us.es
}

Received 15 October 2021; Revised 20 December 2021; Accepted 3 January 2022; Published 28 January 2022

Academic Editor: Nagendra Kumar

Copyright (C) 2022 Vicente Mayor et al. This is an open access article distributed under the Creative Commons Attribution License, which permits unrestricted use, distribution, and reproduction in any medium, provided the original work is properly cited.

\begin{abstract}
Drones equipped with wireless network cards can provide communication services in open areas. This paper proposes a hierarchical two-layered network architecture with two types of drones according to their communication equipment: Access and Distribution. While access drones provide WiFi access to ground users, distribution drones act as WiFi-to-5G relay forwarding packets into the $5 \mathrm{G}$ Core Network. In this context, we formulate a novel optimization problem for the 3-D initial placement of drones to provide Voice over WiFi (VoWiFi) service to ground users. Our optimization problem finds the minimum number of drones (and their type and location) to be deployed constrained to coverage and minimum voice speech quality. We have used a well-known metaheuristic algorithm (Particle Swarm Optimization) to solve our problem, examining the results obtained for different terrain sizes (from $25 \mathrm{~m} \times 25 \mathrm{~m}$ to $100 \mathrm{~m} \times 100 \mathrm{~m}$ ) and ground users (from 10 to 100 ). In the most demanding case, we were able to provide VoWiFi service with four distribution drones and five access drones. Our results show that the overall number of UAVs deployed grows with the terrain size (i.e., with users' sparsity) and the number of ground users.
\end{abstract}

\section{Introduction}

Unmanned Aerial Vehicles (UAVs), also known as drones, have been widely studied in the literature for the last decade partly due to their versatility, which lets UAVs be used in different domains [1]. The application considered in this paper is the UAV-assisted deployment of wireless communication services in open areas $[2,3]$. UAVs equipped with communication electronics have been proposed in the past as mobile base stations or access points to enhance wireless coverage, improve capacity or reliability in existing infrastructure, or even replace damaged infrastructure in emergency situations $[4,5]$.

Provisioning a communication service to ground users typically requires the deployment of a UAV-to-ground access network and, if not in place, a UAV-to-UAV and/or UAV-to-infrastructure network or backhaul [6]. Most research papers tend to focus on only one of these networks and its associated communication technology (e.g., WiMAX [7], cellular 3/4G [8], or 5G [9] for the backhaul and cellular for the access network). Clearly, ground users' devices must share the radio technology in the access network, which could be a problem in practice often overlooked (e.g., scarcity of $5 \mathrm{G}$ users or the involvement of a telco operator). For this reason, some works have also suggested the use of WiFi [10-12] for the UAV-to-ground access network due to its low complexity, ubiquity (e.g., smartphones, tablets and some IoT devices), and independence from operators. UAV-enabled WiFi deployments bring new opportunities when creating provisional communications infrastructure in SAR (Search and Rescue) missions, which is actively investigated in scientific literature nowadays $[12,13]$. However, designing a WiFi access network requires dealing with signal coverage and Quality of Service (QoS) issues at the IEEE 802.11 MAC sublayer. This challenge is translated to the problems addressed in the literature, such as 3-D placement, trajectory planning, energy efficiency, coverage, or backhaul connectivity $[6,14]$.

In this paper, we propose using UAVs to form a twolevel hierarchical network that relays between IEEE 802.11 
(WiFi) and cellular $5 \mathrm{G}$ for providing VoIP over WiFi (VoWiFi) service to ground users (see Figure 1). Access network drones implement WiFi, while distribution/backhaul drones implement a gateway between WiFi and 5G. This combination of technologies leverages the high bandwidth of $5 \mathrm{G}$ (up to $1 \mathrm{Gbps}$ ) to aggregate VoIP traffic flows in a low number of $5 \mathrm{G}$ radio links, reducing the number of mobile subscriptions and costs in $5 \mathrm{G}$ equipment. In this scenario, we address the problem of the initial 3-D placement of the UAVs in both networks. As Masroor et al. put it [4], "the placement of UAVs is an important parameter of resource management as this can affect the transmit power, coverage, and the QoS of the system." More specifically, we propose a new optimization problem that considers constraints about signal coverage and QoS and finds the minimum number of drones that need to be deployed, their position, and type (i.e., access network-A-UAV-or distribution-network-D-UAV). This work extends our previous research $[15,16]$, where we studied the deployment of drones to form a WiFi access network for VoIP services. Now, we integrate the access network into a 5G Core Network using a second layer of drones that hove at a higher altitude and aggregate the VoIP traffic flows. Note that in this scenario, the quality of service depends not only on the congestion level of the access network but also on the distribution network and 5G operator backbone SLAs (Service Level Agreement). As such, UAVs' optimal position may depend on users' location, VoIP capacity, and backbone link capacity.

The main contributions of this paper are as follows:

(i) We mathematically formulate a new problem for the optimal deployment of UAVs that includes coverage and QoS constraints in the access (WiFi), distribution (WiFi), and backbone (5G) networks.

(ii) We propose a novel network reference architecture for the proposed scenario that provides seamless Voice over WiFi (VoWiFi) access to the 5G Core Network.

(iii) We provide an analytical model to estimate the speech quality in our multilayered scenario. Our models assume realistic traffic conditions such as heterogeneous and nonsaturated stations.

(iv) We use a metaheuristic search method (i.e., Particle Swarm Optimization) to solve the previous optimization problem.

The remainder of the paper is as follows. Section 2 presents a state-of-the-art analysis of UAV optimization problems for communications services. Then, Section 3 elaborates on the proposed network architecture and the voice quality assessment method. The proposed optimization problem is defined in Section 4, and Section 5 presents a solution search method based on a well-known meta-heuristics algorithm (Particle Swarm Optimization). Then, we examine the solutions obtained for different scenarios in Section 6 and analyze the performance. Finally, Section 7 summarizes the paper by providing conclusions and future work.

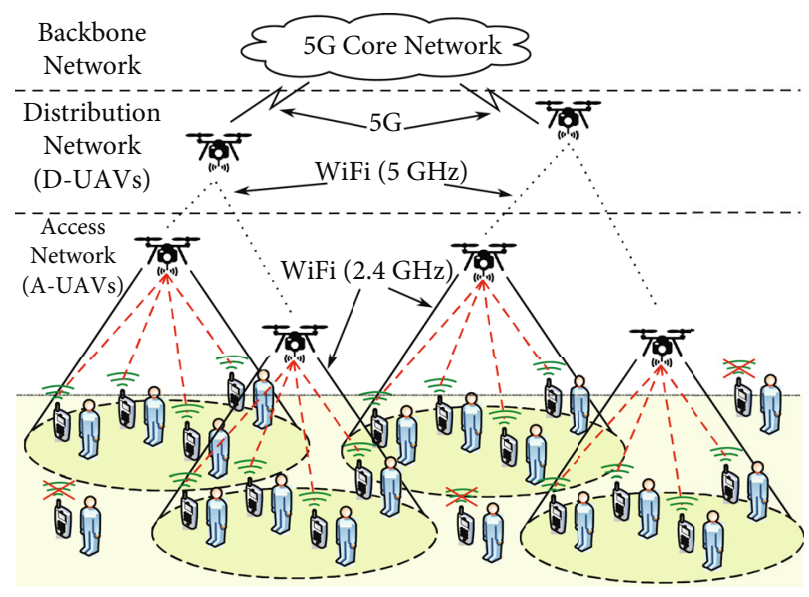

Figure 1: Proposed scenario.

\section{Related Works}

The optimal placement of UAVs has been identified as an open challenge in the context of communication services that use drones as base stations (BS) or relaying nodes in 2D, 3D, single-, and multi-UAV environments [5]. Table 1 summarizes the state of the art in UAV optimal location optimization problems for wireless coverage provisioning and clarifies how our work is different from related works. Works in Table 1 are classified in terms of (a) their objective function, (b) optimization variables (e.g., initial placement, trajectory, or resource allocation), and (c) any other constraints or considerations that might restrict its usage to a concrete scenario.

Optimal UAV placement for wireless coverage counts with a good deal of optimization problems in the literature. For example, Li et al. [17] propose to maximize throughput via power allocation and $3 \mathrm{D}$ placement for indoor communications. Maximizing the throughput has also been addressed in [18], where the authors suggest a 3D UAV placement and power allocation optimization problem based on softwaredefined cellular networks, or in [19] that also employs software-defined cellular networks but using rate instead of power allocation. Similarly, Yin et al. [20] suggest deploying a cellular network (FDMA) by maximizing the downlink rate under three optimization variables: user association, resource allocation, and UAV placement. Other authors [21] suggest combining UAV placement with user association in order to minimize the maximum traffic demand at UAVs, achieving a fair traffic distribution among them; or the deployment of NOMA (Nonorthogonal Multiple Access) networks to mitigate the path loss (i.e., maximize received signal strength) or energy consumption due to transmit power [22, 23], respectively. Finally, in $[24,25]$, the authors propose to minimize the number of deployed UAVs while finding the positioning that maximizes the coverage area while forming a robust backbone network.

Besides optimal initial positioning, trajectory optimization (i.e., a series of discrete points of a flying path that drones follow in a continuous movement while providing wireless network coverage [4]) has also been widely 
TABLE 1: Related work comparison.

\begin{tabular}{|c|c|c|c|c|c|c|c|c|c|c|}
\hline \multirow[t]{2}{*}{ Ref. } & \multirow[t]{2}{*}{ Objective function } & \multicolumn{3}{|c|}{ Variables } & \multicolumn{2}{|c|}{$\begin{array}{l}\text { Network } \\
\text { technologies }\end{array}$} & \multicolumn{4}{|c|}{ Considerations } \\
\hline & & Placement & Trajectory & $\begin{array}{l}\text { Resource } \\
\text { allocation }\end{array}$ & Access & Backbone & SINR & BW & $\begin{array}{l}\text { Speech } \\
\text { quality }\end{array}$ & Energy \\
\hline [17] & max. throughput & $\checkmark$ & $x$ & $x$ & FDMA & FDMA & $\checkmark$ & $x$ & $x$ & $x$ \\
\hline [18] & max. throughput & $\checkmark$ & $x$ & $\checkmark$ & Cellular & Cellular & $\checkmark$ & $x$ & $x$ & $\checkmark$ \\
\hline [19] & max. throughput & $\checkmark$ & $x$ & $\checkmark$ & Cellular & Cellular & $\checkmark$ & $\checkmark$ & $x$ & $x$ \\
\hline [20] & max. downlink rate & $\checkmark$ & $x$ & $\checkmark$ & Cellular & Cellular & $\checkmark$ & $x$ & $x$ & $x$ \\
\hline [21] & $\begin{array}{l}\text { min. maximum traffic } \\
\text { demand }\end{array}$ & $\checkmark$ & $x$ & $\checkmark$ & Cellular & Cellular & $\checkmark$ & $x$ & $x$ & $x$ \\
\hline [22] & min. path loss & $\checkmark$ & $x$ & $\checkmark$ & NOMA & Cellular & $\checkmark$ & $x$ & $x$ & $\checkmark$ \\
\hline [23] & $\begin{array}{l}\text { min. transmit power or } \\
\text { max. rate }\end{array}$ & $\checkmark$ & $x$ & $\checkmark$ & NOMA & Cellular & $\checkmark$ & $x$ & $x$ & $\checkmark$ \\
\hline [24] & $\begin{array}{l}\text { min. UAVs and max. } \\
\text { coverage }\end{array}$ & $\checkmark$ & $\checkmark$ & $\checkmark$ & Cellular & Cellular & $\checkmark$ & $\checkmark$ & $x$ & $x$ \\
\hline [25] & $\begin{array}{l}\min \text {. UAVs and max. } \\
\text { coverage }\end{array}$ & $\checkmark$ & $\checkmark$ & $\checkmark$ & Cellular & Cellular & $\checkmark$ & $\checkmark$ & $x$ & $x$ \\
\hline [26] & max. energy efficiency & $x$ & $\checkmark$ & $x$ & mmWave & Cellular & $\checkmark$ & $x$ & $x$ & $\checkmark$ \\
\hline [27] & min. power allocation & $x$ & $\checkmark$ & $\checkmark$ & OFDMA & OFDMA & $\checkmark$ & $x$ & $x$ & $\checkmark$ \\
\hline [28] & min. energy consumption & $x$ & $\checkmark$ & $\checkmark$ & Cellular & Cellular & $\checkmark$ & $x$ & $x$ & $\checkmark$ \\
\hline [29] & $\begin{array}{l}\text { max. spectrum and energy } \\
\text { eff. }\end{array}$ & $x$ & $\checkmark$ & $\checkmark$ & TDD & TDD & $x$ & $x$ & $x$ & $\checkmark$ \\
\hline [30] & $\begin{array}{l}\text { max. spectrum and energy } \\
\text { eff. }\end{array}$ & $x$ & $\checkmark$ & $\checkmark$ & TDD & TDD & $x$ & $x$ & $x$ & $\checkmark$ \\
\hline [31] & max. minimum throughput & $x$ & $\checkmark$ & $\checkmark$ & Cellular & Cellular & $x$ & $\checkmark$ & $x$ & $x$ \\
\hline [32] & max. minimum user rate & $x$ & $\checkmark$ & $\checkmark$ & OFDMA & OFDMA & $\checkmark$ & $x$ & $x$ & $x$ \\
\hline [33] & max. minimum user rate & $x$ & $\checkmark$ & $\checkmark$ & TDMA & TDMA & $\checkmark$ & $x$ & $x$ & $\checkmark$ \\
\hline [34] & $\begin{array}{l}\text { max. end-to-end } \\
\text { throughput }\end{array}$ & $x$ & $\checkmark$ & $\checkmark$ & Cellular & Cellular & $\checkmark$ & $x$ & $x$ & $\checkmark$ \\
\hline [35] & $\begin{array}{l}\text { max. end-to-end } \\
\text { throughput }\end{array}$ & $x$ & $\checkmark$ & $\checkmark$ & Cellular & Cellular & $\checkmark$ & $x$ & $x$ & $\checkmark$ \\
\hline [36] & min. outage probability & $x$ & $\checkmark$ & $\checkmark$ & Cellular & Cellular & $\checkmark$ & $x$ & $x$ & $\checkmark$ \\
\hline [15] & $\begin{array}{l}\text { min. UAVs and user radio } \\
\text { energy }\end{array}$ & $\begin{array}{l}\checkmark \text { (single- } \\
\text { layer) }\end{array}$ & $x$ & $\checkmark$ & WiFi & $\mathrm{N} / \mathrm{A}$ & $\checkmark$ & $\checkmark$ & $\checkmark$ & $\checkmark$ \\
\hline [16] & $\begin{array}{l}\text { min. UAVs and UAVs' } \\
\text { energy }\end{array}$ & $\begin{array}{l}\checkmark \text { (single- } \\
\text { layer) }\end{array}$ & $x$ & $\checkmark$ & WiFi & $\mathrm{N} / \mathrm{A}$ & $\checkmark$ & $\checkmark$ & $\checkmark$ & $\checkmark$ \\
\hline $\begin{array}{l}\text { This } \\
\text { paper }\end{array}$ & $\begin{array}{l}\text { min. UAVs and user } \\
\text { coverage }\end{array}$ & $\begin{array}{c}\checkmark \\
\text { (multilayer) }\end{array}$ & $x$ & $\checkmark$ & WiFi & $5 \mathrm{G}$ & $\checkmark$ & $\checkmark$ & $\checkmark$ & $x$ \\
\hline
\end{tabular}

addressed, mainly focusing on energy efficiency. Chakareski et al. [26] proposed an energy-efficient framework for deploying mmWave $5 G$ cellular networks, in $[27,28]$, the authors proposed a joint optimization problem for trajectory and power allocation, and finally, $[29,30]$ present a joint spectrum and energy efficiency optimization problem. Through put maximization optimization via trajectory has also been proposed in several works. Hu et al. [31] proposed a resource and trajectory optimization problem by introducing user scheduling and bandwidth allocation; and $[32,33]$ proposed to combine trajectory with radio resource allocation in vehicular and UAV-enabled relaying systems. Finally, other works such as $[34,35]$ proposed to maximize the end-to-end rate by considering trajectory power allocation optimization while deploying multi-hop relay networks. Finally, [36] proposed to minimize the probability of outage through a joint power allocation and trajectory optimization problem.

Few works have addressed the provision of VoIP service with drones. To the best of our knowledge, only our previous work $[15,16]$ has done it considering a single-layer of drones to set up a WiFi access network. In both papers, radio coverage and speech quality constrained the $3 \mathrm{D}$ multi-UAV placement optimization problem, seeking the minimum deployment costs (i.e., number of UAVs deployed). Between solutions with the same number of drones, we chose the one that minimized users' radio energy or UAVs energy, respectively. However, both works addressed only the WiFi access network, leaving the distribution and backbone networks for future research. This paper takes a step further by 
considering a two-layered hierarchical network that performs access, distribution, and connection to the backbone using two different radio technologies: $\mathrm{WiFi}$ and 5G. Regarding the objective function, we propose to find the minimum number of deployed UAVs (independently of their type) needed to maximize user coverage. In light of Table 1, we can state that our work differs from those found in the literature in three main aspects: (a) a multilayered UAV placement that performs traffic aggregation, (b) more realistic constraints for real-time voice speech quality by modelling the MAC sublayer of the access network, and (c) the deployment of a WiFi access network instead of a cellular-based one, hence minimizing any compatibility issues regarding user smartphones.

\section{Proposed Scenario and QoS Assessment}

As shown in Figure 1, our scenario consists of a two-level hierarchical network that relays between IEEE 802.11 (WiFi) networks (i.e., for access and distribution) and cellular 5G backhaul links. The QoS experienced by users will be influenced by packet loss and delay in these networks. This section is devoted to defining the network architecture and the analytical model used to estimate the speech quality experienced by users in our scenario.

3.1. Network Architecture. Figure 2 illustrates the proposed network architecture. The network entities (from left to right) are as follows:

(i) Ground users are equipped with WiFi-compliant terminals (e.g., smartphones) operating at the
$2.4 \mathrm{GHz}$ band associated to the Access Point installed at A-UAVs.

(ii) A-UAVs play a double role: (a) for users, they act as access points (at the $2.4 \mathrm{GHz}$ band), and (b) they are associated with the distribution (second) layer of drones through another WiFi connection at $5 \mathrm{GHz}$.

(iii) D-UAVs behave as WiFi APs for associated AUAVs, and they also provide a backbone link to the 5G network. As such, D-UAVs are seen as 5G User Equipment (UE) maintaining a connection to the 5G Core Network (i.e., GTP-U tunnels).

In the previous architecture, the speech quality experienced by users is impaired on each subnetwork due to the medium access control mechanism and network congestion. The following section elaborates on the proposed QoS assessment method considered in this paper (E-Model).

3.2. Voice Quality Assessment. Voice quality assessment has been studied during the last decades [37]. Some of the approaches suggested in the literature to measure or estimate speech quality, such as P.863 or P.563, require invasive monitoring (e.g., sampling the original voice signal). On the contrary, the E-model [38] is an analytical model that allows one to estimate the speech quality assuming additive impairments to the quality. The E-model provides a quality score, namely, the $R$ factor, that ranges from 0 (poor) to 100 (excellent) that is calculated as follows $[15,39,40]$ :

$$
R=R_{0}-\underbrace{I_{e}+\left(95-I_{e}\right) \frac{P_{p l}}{\left(P_{p l} / \text { Burst } R\right)+B_{p l}}}_{I_{e, e f f}}-\underbrace{(0.024 d+0.11 \cdot(d-177.3) \cdot H(d-177.3))}_{I_{d}} .
$$

In the previous equation, two impairments $\left(I_{e, \text { eff }}\right.$ and $\left.I_{d}\right)$ are subtracted from the maximum achievable quality $\left(R_{0}\right)$, which is a representation of the Signal-to-Noise Ratio.

$I_{e, \text { eff }}$ represents a combination between impairment equipment parameter at zero packet loss $\left(I_{e}\right)$ and a function that depends on $I_{e}$, the packet loss rate, and packet loss behavior. $I_{e}$ is a codec-dependent constant associated with codec compression degradation (a list of values from ITU$\mathrm{T}$ codecs were presented in ITU-T Rec. G.113 Appendix I), $B_{\mathrm{pl}}$ represents the codec packet loss robustness, which also has a specific value for each codec (listed in ITU-T Rec. G117 Appendix I), $P_{\mathrm{pl}}$ represents the packet loss rate (in $\%)$ in the WiFi channel, and finally, Burst $R$ characterizes the burst ratio (i.e., equals 1 if the packet loss is random and greater otherwise). In this paper, we use the G.711 codec $\left(I_{e}=0\right.$ and $\left.B_{\mathrm{pl}}=25.1\right)$ and assume random losses (i.e., Burst $R=1)$.
The latest impairment, $I_{d}$, is associated with the delay in the communication chain. A widely accepted approximation for $I_{d}$ can be obtained from one-way delay in the communication path $d$, where $H$ is the Heaviside function $(H(x)=0$ for $x<0$ and $H(x)=1$ for $x>0$. In our case, $x=d-177.3$. The one-way delay $d$ (in milliseconds) includes all additive delays from source to destination, which depend on the network topology and the chosen codec (i.e., packetization delay).

In order to solve Equation (1), one has to model both network performance parameters (i.e., network delay $d$ and the packet loss rate $P_{\mathrm{pl}}$ ). Since our scenario consists of WiFi access and distribution networks and a backhaul 5G link, the overall delay and packet loss ratio should be

$$
P_{\mathrm{pl}}=1-\left(\left(1-P_{\mathrm{pl}, a}\right)\left(1-P_{\mathrm{pl}, d}\right)\left(1-P_{\mathrm{pl}, b}\right)\right) \text {, }
$$




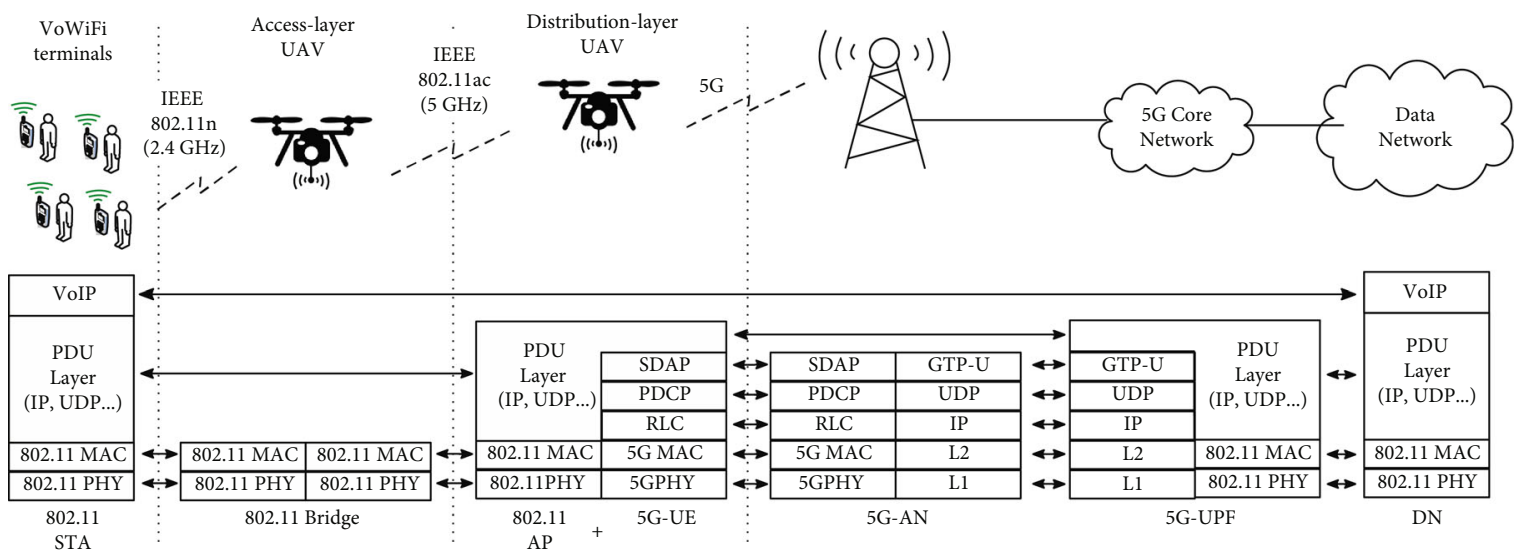

FIGURE 2: Network reference architecture.

$$
d=d_{p}+d_{a}+d_{d}+d_{b}
$$

where $(a),(d)$, and $(b)$ subscripts refer to impairments at the access, distribution (i.e., influenced by IEEE 802.11 physical and MAC sublayer), and backhaul link, respectively. Finally $d_{p}$ accounts for the packetization delay, which could be assumed as a constant (e.g., $20 \mathrm{~ms}$ for G.711 codec).

The previous performance metrics can be estimated for the IEEE 802.11 network through well-known analytical models (see $[15,16])$ that enable the prediction of the packet loss ratio and delay in the WiFi network just by defining the number of stations, traffic models (i.e., packet size and frequency), and stations' received signal strength. On the other hand, 5G links performance can be assumed to be included in the Service Level Agreement with the telco provider [41], so one can easily obtain $P_{\mathrm{pl}, b}$ and $d_{b}$.

\section{Problem Definition}

In this section, we state the terminology, main assumptions, and a formal definition of the problem of optimal placement under the scenario illustrated in Figures 1 and 2.

4.1. Terminology. Table 2 summarizes the notation used in this paper. We use three sets to represent distribution drones $(\mathscr{D})$, access drones $(\mathscr{A})$, and ground users $(\mathcal{U})$. The location of each element in these sets is represented in Cartesian's coordinates: $\vec{d}, \vec{a}$, and $\vec{u}$, respectively.

Let us introduce some functions that will be recurrently used in this paper:

(i) The coverage function, $\mathscr{C}(\vec{x})$, represents the set of stations associated with a specific UAV-mounted AP identified by its location, e.g. $\vec{x}$. For example:

(a) $\mathscr{C}\left(\vec{d}_{i}\right)$ represents the set of A-UAVs associated with the $i$-th D-UAV. (b) $\mathscr{C}\left(\vec{a}_{j}\right)$ represents the set of users associated with the $j$-th A-UAV.

(c) $C=\sum_{i=1}^{A}\left|\mathscr{C}\left(\vec{a}_{i}\right)\right|$ represents the number of users covered by the system (and hence associated to any A-UAV).

(ii) The speech quality function, $R\left(\vec{d}_{i}, \vec{a}_{j}\right)$, provides the speech quality experience by users associated with the $j$-th A-UAV (see Equation (3)) which in turn is associated with the $i$-th D-UAV. This function takes into account the level of congestion in the access and distribution networks

(iii) The uplink throughput function, $S\left(\vec{d}_{i}\right)$, provides the aggregated throughput (in $\mathrm{Mb} / \mathrm{s}$ ) handled by the $i$ -th D-UAV. Clearly, it cannot exceed the maximum throughput achievable by the $5 \mathrm{G}$ link $\left(S_{\max }\right)$.

4.2. Assumptions. For the sake of tractability, the following assumptions are made:

(i) Ground users are confined in a flat outdoor terrain with known dimensions. Their position is also known (this could be implemented through users' smartphone's GPS or image processing).

(ii) Users' calls go through a compatible VoIP application that uses a known audio codec.

(iii) APs' channelization is arranged in such a manner that the interference between adjacent radio channels is negligible. By using directional antennas, one could simply adjust the radio beam pattern to minimize inter-UAV interference.

(iv) Ground users could potentially be simultaneously on a call. Thus, each A-UAV will not have more users associated than its VoIP Capacity [42] (maximum number of simultaneous calls under a guaranteed QoS). Then, VoIP Capacity is a harder constraint than coverage and will play a 
TABLE 2: System terminology summary.

\begin{tabular}{lcc}
\hline Symbol & Example & Description \\
\hline $\mathscr{D}$ & $\left\{\vec{d}_{1}, \vec{d}_{2}, \cdots, \vec{d}_{D}\right\}$ & Set of D-UAVs' locations \\
$D$ & $|\mathscr{D}|$ & Number of deployed D-UAVs \\
$\vec{d}_{i}$ & $\left\{x_{i}, y_{i}, z_{i}\right\}$ & Cartesian coordinates of the $i$-th D-UAV \\
$\mathscr{A}$ & $\left\{\vec{a}_{1}, \vec{a}_{2}, \cdots, \vec{a}_{A}\right\}$ & Set of A-UAVs' locations \\
$A$ & $|\mathscr{A}|$ & Number of deployed A-UAVs \\
$\vec{a}_{j}$ & $\left\{x_{j}, y_{j}, z_{j}\right\}$ & Cartesian coordinates of the $j$-th A-UAV \\
$\mathcal{U}$ & $\left\{\vec{u}_{1}, \vec{u}_{2}, \cdots, \vec{u}_{U}\right\}$ & Set of ground users' locations \\
$U$ & $|\mathcal{U}|$ & Number of ground users \\
$\vec{u}_{k}$ & $\left\{x_{k}, y_{k}, z_{k}\right\}$ & Cartesian coordinates of the $k$-th user \\
\hline
\end{tabular}

decisive role in determining the number of $\mathrm{A}$ UAVs deployed.

4.3. Optimization Problem. The formal definition of our optimization problem is stated in

$$
\begin{aligned}
& \min _{\mathscr{D}, \mathscr{A}}(D+A)+\left(1-\frac{C}{U}\right) \\
& \text { subject to } \quad \frac{C}{U} \geq C_{\min } \\
& R\left(\vec{d}_{i}, \vec{a}_{j}\right) \geq R_{\min }, \quad \forall \vec{d}_{i} \in \mathscr{D}, \vec{a}_{j} \in \mathscr{C}\left(\vec{d}_{i}\right) \\
& S\left(\vec{d}_{i}\right) \leq S_{\max }, \quad \forall \vec{d}_{i} \in \mathscr{D}, \\
& \vec{d}_{i} \in \mathscr{X}_{D}, \quad \forall \vec{d}_{i} \in \mathscr{D}, \\
& \vec{a}_{i} \in \mathscr{X}_{A}, \quad \forall \vec{a}_{i} \in \mathscr{A}, \\
& D \leq D_{\max } \\
& A \leq A_{\text {max }} \text {, }
\end{aligned}
$$

where $\mathscr{X}_{D}$ and $\mathscr{X}_{A}$ stand for the collection of valid positions for distribution and access UAVs, respectively; so one could restrict flying zones or establish a range of valid altitudes for each type of UAV.

The previous expression optimizes the location of Distribution and Access UAVs $(\mathscr{D}, \mathscr{A}$, respectively) that minimizes the total number of UAVs deployed $(D+A)$ while maximizing user coverage $(C / U)$ subject to the following constraints:

(i) User coverage ratio, $C / U$, must exceed a threshold $\left(C_{\min }\right)$.

(ii) Offered speech quality, $R\left(\vec{d}_{i}, \vec{a}_{j}\right) \forall \vec{d}_{i} \in \mathscr{D}, \vec{a}_{j} \in \mathscr{C}$

\section{$\left(\vec{d}_{i}\right)$, must be greater than a threshold $\left(R_{\min }\right)$.}

(iii) D-UAVs uplink throughput, $S\left(\vec{d}_{i}\right) \forall \vec{d}_{i} \in \mathscr{D}$, must be lower than $5 \mathrm{G}$ links capacity $\left(S_{\max }\right)$.

Observe that the analytical expression in Equation (4) is composed of two terms: $(D+A)$ which is an integer (always $\geq 2$ since we need to deploy at least one drone of each kind) and $(1-C / U)$ which represent the rate of uncovered users. The latter is a real number always less than 1 (e.g., a coverage of $80 \%$ would provide 0.2 ) and, as such, is subordinated to the first (which is an integer). So minimizing the number of drones in the solution always takes precedence over coverage (which is already a problem constraint). Thus, the second term is only used to break the deadlock between various solutions with the same number of drones (the one with greater coverage is preferred).

\section{Problem Resolution}

The optimization problem in (4) can be solved through metaheuristics search algorithms. In our previous work $[15,16]$, we demonstrated the effectiveness of Genetic Algorithm and Particle Swarm Optimization, comparing their results with exhaustive search. For simplicity's sake, and given our previous experience, we will use a PSO algorithm (further proposed by other authors [43]) in this paper.

5.1. Search Algorithm. Algorithm 1 contains the pseudocode of the search algorithm used to solve the optimization problem in (4). The input of the algorithm is the set of valid positions for UAVs $(\mathscr{X})$, the set of ground users' location $(\mathscr{U})$, and the problem constraints $\left(D_{\max }, C_{\min }, R_{\min }, S_{\max }\right)$. This algorithm looks for the optimal location of UAVs $(\mathscr{D}, \mathscr{A})$ that meets the problem constraint and increases the number of drones $(A$, and $D)$ until a valid solution is found. For a given number of drones $(D \leq A)$, their positioning is jointly optimized by the SolveOptim_PSO function.

On each iteration, the SolveOptim_PSO (see Algorithm 2) is called to find the optimal location $\left(\mathscr{D}^{*}, \mathscr{A}^{*}\right)$ for 


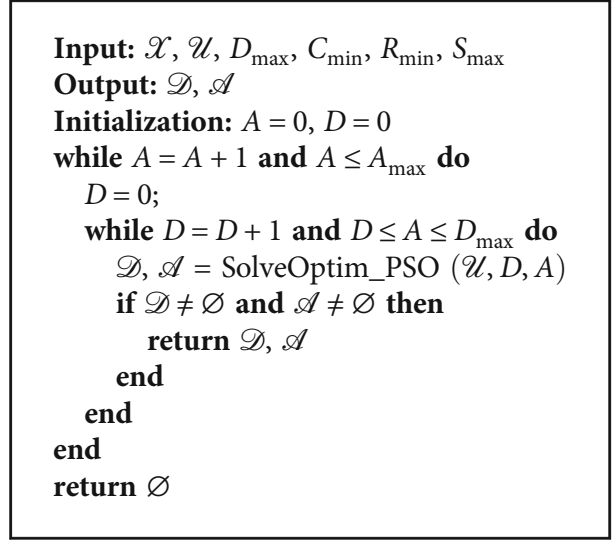

Algorithm 1: Search algorithm pseudocode.

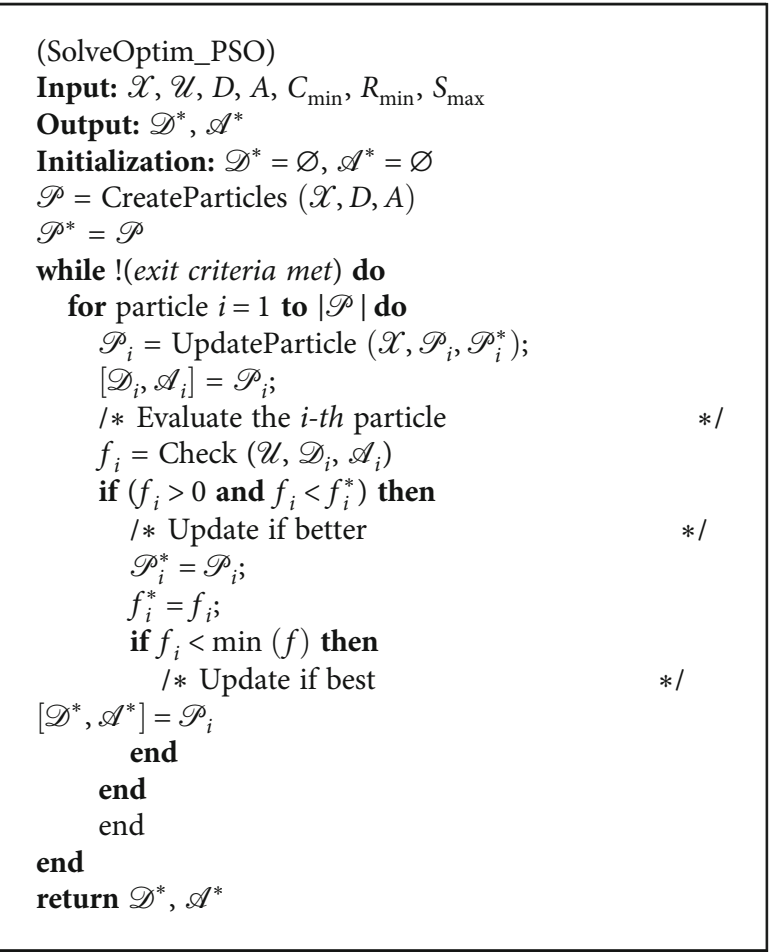

Algorithm 2: Particle Swarm Optimization function

the specified number of UAVs (i.e., $D$ and $A$ input parameters), returning an empty set if the problem constraints cannot be met. During execution, candidate locations (i.e., particles) are randomly created (CreateParticles), evolved (UpdateParticle), and evaluated (Check) following a common Particle Swarm Optimization approach:

(i) The CreateParticles function returns a set of particles randomly generated, $\mathscr{P}$, by considering the collection of valid positions $(\mathscr{X})$ and the number of Distribution and Access UAVs, $D$ and $A$, respectively. Each element in $\mathscr{P}$ represents a candidate set of locations for all UAVs, so they can be decomposed into the $\mathscr{D}$ and $\mathscr{A}$ sets (ii) The UpdateParticle function updates the set of particles $(\mathscr{P})$ by considering the best-found location for each particle $\left(\mathscr{P}^{*}\right)$

(iii) The Check function contains the fitness evaluation that returns a score for a given location. Then, the algorithm evaluates if the candidate position is valid $\left(f_{i}>0\right)$, and if it improves the best-found location for that particle $\left(f_{i}<f_{i}^{*}\right)$. If so, the best-found location for that particle is updated

(iv) The algorithm finishes when the exit criterion is achieved (e.g., the overall improvement between two consecutive generations is lower than $10^{-3}$ ). It returns the location for Distribution and Access UAVs $\left(\mathscr{D}^{*}\right.$ and $\left.\mathscr{A}^{*}\right)$ if any, or an empty set otherwise

The next section elaborates on the fitness evaluation procedure (Check function); the reader can find rest of the PSO-related functions in our previous work [16], along with some implementation details.

5.2. Fitness Evaluation (Check Function). For every candidate solution (i.e., $\mathscr{D}, \mathscr{A}$ ), a fitness score has to be calculated based on the optimization problem. Let us define the score of a particle (i.e., candidate solution) as $f$ :

$f=\left\{\begin{array}{l}(D+A)+\left(1-\frac{C}{U}\right), \quad \text { if constraints are satisfied, } \\ -\frac{C}{U}, \quad \text { otherwise, }\end{array}\right.$

where constraints refers to coverage, quality of service, and throughput evaluation.

5.2.1. Coverage Evaluation. Checks if the total number of covered users $(C)$ is greater than the threshold $\left(C_{\min }\right)$. It uses the coverage function, $\mathscr{C}\left(\vec{x}_{i}\right)$, that returns a set of locations of associated Stations for an Access Point located at $\vec{x}_{i}$ (i.e., in Cartesian coordinates).

This function can be applied to any AP in the system (i.e., both D-UAVs and A-UAVs). For example, (6) represents the coverage function for the $i$-th D-UAV $\left(\vec{d}_{i}\right)$, but it can be adapted for any A-UAV just by replacing $\vec{d}$ and $\vec{a}$ with $\vec{a}$ and $\vec{u}$, respectively.

$$
C\left(\vec{d}_{i}\right)=\left\{\begin{array}{l|l}
\operatorname{RSSI}\left(\vec{d}_{i}, \vec{\alpha}_{k}\right) \geq R S S I_{\min } \\
\vec{\alpha}_{k} \in \mathscr{A} & \operatorname{SINR}\left(\vec{d}_{i}, \vec{\alpha}_{k}\right) \geq S I N R_{\min } \\
\operatorname{RSSI}\left(\vec{d}_{i}, \vec{\alpha}_{k}\right)>\operatorname{RSSI}\left(\vec{d}_{i}, \vec{\alpha}_{j}\right) \forall \vec{\alpha}_{k} \neq \vec{\alpha}_{j}
\end{array}\right\}
$$


The previous expression returns the subset of stations that (a) are compliant with RSSI and SINR minimum thresholds and (b) have the greatest signal intensity. Then, RSSI and SINR can be calculated as follows.

(i) The Received Signal Strength Indicator (RSSI) depends on the distance and angle between the transmitter and the receptor. Equation (7) represents the RSSI calculus (in $\mathrm{dBm}$ ). In this expression, an initial transmission power is considered $\left(P_{\mathrm{tx}}\right)$, further adding power gains $(G)$ and subtracting any losses $(L)$.

$$
\begin{gathered}
\operatorname{RSSI}(\vec{x}, \vec{y})=P_{\mathrm{tx}}+G\left(\theta_{x, y}\right)-L\left(\|\vec{x}-\vec{y}\|, \theta_{x, y}\right), \\
G(\theta)=10 \log _{10}\left(10^{G_{\max } / 20} \cdot \cos ^{2} \theta\right), \\
L(d, \theta)=P_{\mathrm{LoS}}(\theta) L_{\mathrm{LoS}}(d)+P_{\mathrm{NLoS}}(\theta) L_{\mathrm{NLoS}}(d),
\end{gathered}
$$

where 8 represent a simple gain pattern of an antenna (limited by $G_{\max }$ ) and $L$ represents a path loss expression widely proposed in air-to-ground channel models [44-46]. It considers that ground users receive three different groups of signals: (a) Line-of-Sight (LoS), (b) Non-Line-of-Sight (NLoS), and (c) other reflected components which cause multipath fading. Each group has its own probability of occurrence depending on the environment, density, height, and elevation angle. According to [47], the probability of fading is significantly lower than the probability of receiving the LoS $\left(P_{\mathrm{LoS}}\right)$ and $\mathrm{NLoS}\left(P_{\mathrm{NLoS}}\right)$ components, so we will neglect it for simplicity. $L_{\mathrm{LoS}}$ and $L_{\mathrm{NLoS}}$ represent the average path loss for LoS and NLoS components, respectively, and can be expressed as [44]

$$
\begin{gathered}
L_{\mathrm{LoS}}(d)=20 \log _{10}\left(\frac{4 \pi f_{c} d}{c}\right)+\xi_{\mathrm{LoS}} \\
L_{\mathrm{NLoS}}(d)=20 \log _{10}\left(\frac{4 \pi f_{c} d}{c}\right)+\xi_{\mathrm{NLoS}}
\end{gathered}
$$

where the first term accounts for the free space propagation loss $\xi_{\mathrm{LoS}}$ and $\xi_{\mathrm{NLoS}}$ represent any additional losses due to the environment (a list of values can be found in [45]) and $f_{c}$ is the channel frequency.

Finally, the probability of receiving each component is given by [48]

$$
\begin{gathered}
P_{\mathrm{LoS}}(\theta)=\frac{1}{1+\alpha e^{-\beta \theta \cdot 180 / \pi+\beta \alpha}}, \\
P_{\mathrm{NLoS}}(\theta)=1-P_{\mathrm{LoS}}(\theta),
\end{gathered}
$$

where $\alpha$ and $\beta$ are environment-dependent constants (e.g., rural and urban) as defined in [45] (e.g., $\alpha=\beta=0$ for no buildings).

(i) The Signal-to-Interference-plus-Noise-Ratio (SINR) can be calculated by subtracting every noise and cochannel interference from the received signal
TABLE 3: Input parameters.

\begin{tabular}{lc}
\hline Parameter & Value \\
\hline Users & 60 \\
Size & $100 \mathrm{~m} \times 100 \mathrm{~m}$ \\
VoIP codec & G.711 $(20 \mathrm{~ms}$ interval $)$ \\
D-UAVs' WiFi & $802.11 \mathrm{ac}$ at $5 \mathrm{GHz}(80 \mathrm{MHz})$ \\
A-UAV' WiFi & $802.11 \mathrm{n}$ at $2.4 \mathrm{GHz}(20 \mathrm{MHz})$ \\
D-UAVs' altitude & {$[10,35] \mathrm{m}$} \\
A-UAVs' altitude & {$[40,55] \mathrm{m}$} \\
RSSI $_{\text {min }}$ & $-82 \mathrm{dBm}$ \\
SNR $_{\text {min }}$ & $20 \mathrm{~dB}$ \\
$R_{\text {min }}$ & 65 \\
$C_{\text {min }}$ & $90 \%$ \\
$L_{5 \mathrm{G}-S L A}$ & $1 \%$ \\
$d_{5 \mathrm{G}-S L A}$ & $5 \mathrm{~ms}$ \\
$S_{\text {max }}$ & $1 \mathrm{~Gb} / \mathrm{s}$ \\
\hline
\end{tabular}

strength. Let $\mathscr{Z}$ be the collection of APs operating at an overlapping channel (channel planning is out of the scope of this paper). Then, SINR can be expressed as

$$
\operatorname{SINR}=\frac{P_{r x}}{N+\sum_{k \in \mathscr{Z}} I_{k}}
$$

where $P_{r x}$ represents the received signal power, $N$ accounts for the noise, and each $I_{k} \forall k \in \mathscr{Z}$ stands for the received interference from any overlapping transmissions. Since we assume that the sources of interference are negligible due to a correct channel planning, SINR can be reduced to SNR and it can be calculated as

$$
\begin{gathered}
\left.\operatorname{SINR}\right|_{\mathrm{dB}}(\vec{x}, \vec{y})=\operatorname{RSSI}(\vec{x}, \vec{y})-F-\left.N\right|_{\mathrm{dB}}, \\
\left.N\right|_{\mathrm{dB}}=-174+10 \log _{10}\left(C_{\mathrm{BW}}\right),
\end{gathered}
$$

where $N$ is the noise, $F$ accounts for the receiver's noise figure, and $C_{\mathrm{BW}}$ stands for the signal bandwidth, which depends on the IEEE 802.11 standard revision under consideration.

5.2.2. Speech Quality and Throughput Evaluation. The goal of this evaluation is to guarantee that (a) the speech quality, given by the QoS function $R\left(\vec{d}_{i}, \vec{a}_{j}\right)$ for every D-UAV-A$\mathrm{UAV}$ association in the system, is over a minimum threshold $\left(R_{\min }\right)$ and (b) that the aggregated throughput at D-UAVs do not exceed the maximum capacity of $5 \mathrm{G}$ links $\left(S_{\max }\right)$. Both constraints can be formally represented as (17) and (18), respectively.

$$
R\left(\vec{d}_{i}, \vec{a}_{j}\right) \geq R_{\min }, \quad \forall \vec{d}_{i} \in \mathscr{D}, \forall \vec{a}_{j} \in \mathscr{C}\left(\vec{d}_{i}\right),
$$




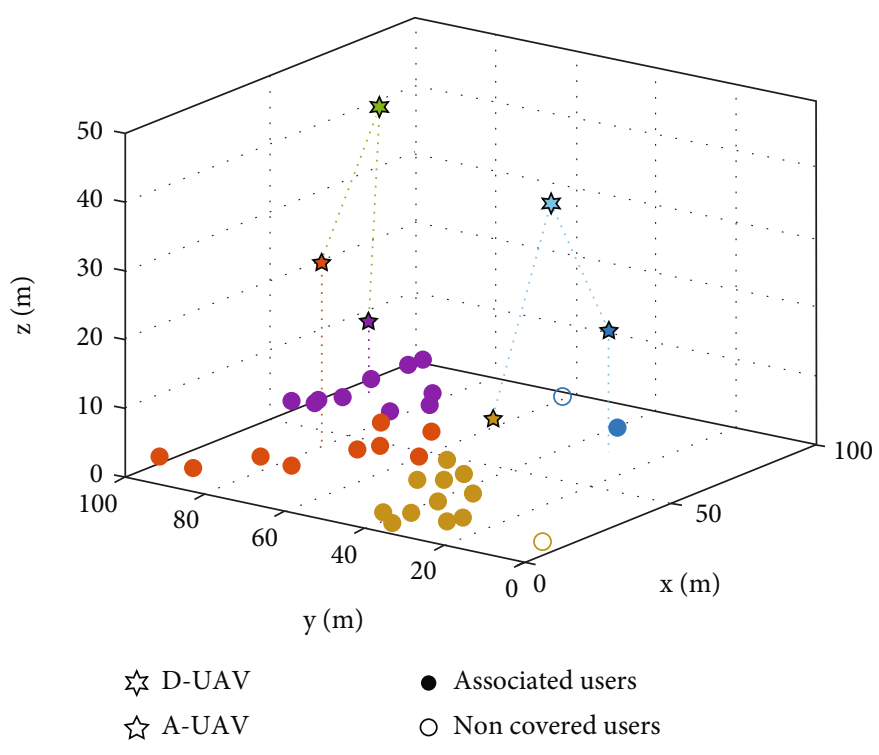

FIGURE 3: Example solution.

$$
S\left(\vec{d}_{i}\right) \leq S_{\max }, \quad \forall \vec{d}_{i} \in \mathscr{D}
$$

To verify these constraints, one has to estimate end-toend performance parameters such as packet loss ratio, delay, and effective throughput. Then, as introduced in Section 3.2, the end-to-end speech quality (e.g., see $R$ factor in (1)) can be estimated through the E-model. This requires to know the end-to-end packet loss ratio $\left(P_{\mathrm{pl}}\right)$ and delay $(d)$. Moreover, $P_{\mathrm{pl}}$ and $d$ can be further decomposed (see Equations (2) and (3)) considering the three networks traversed in the proposed network architecture: (a) the WiFi access network, (b) the WiFi distribution network, and (c) the 5G link SLA. Notice that the delay and loss in the $5 \mathrm{G}$ link can be agreed with the service provider (i.e., the telco operator) and hence can be seen as constant values. However, the first two (WiFi networks) have to be estimated.

To derive the VoIP Capacity of any AP in our system, we extend Bianchi's Markov-chain analytical model [49] of the IEEE 802.11 MAC sublayer performance. Our analytical model takes the location of the access point (e.g., $\vec{a}$ ) and its associated stations $(\mathscr{C}(\vec{a}))$ as input parameters and returns a $\tau$ expression for each contending station as its probability of transmission attempt. The output of our analytical model can be used to derive the estimated delay, packet loss ratio, and aggregated throughput at a given AP as follows:

$$
\begin{gathered}
L_{\mathrm{WiFi}}(\vec{a})=1-\frac{\left(1-\mathrm{FER}^{(\vec{a})}\right) \tau^{(\vec{a})} \prod_{\vec{u} \in \mathscr{C}(\vec{a})}\left(1-\tau^{(\vec{u})}\right)}{|\mathscr{C}(\vec{a})| \cdot \lambda \cdot E[T]} \\
D_{\mathrm{WiFi}}(\vec{a})=E\left[B^{(\vec{a})}\right] \cdot E[T]
\end{gathered}
$$

$$
S_{\mathrm{WiFi}}(\vec{a})=\sum_{\vec{u} \in \mathscr{C}(\vec{a})}\left(\frac{\left(1-F E R^{(\vec{u})}\right) \tau^{(\vec{u})} \prod_{\vec{i} \neq \vec{u} \in \mathcal{S}}\left(1-\tau^{(\vec{i})}\right)}{E[T]}\right) \cdot L_{\mathrm{PDU}}
$$

where $\operatorname{FER}^{(\vec{a})}$ stands for the average Frame Error Rate at the access point, which can be derived from its SINR and Modulation and Coding Scheme (MCS); $E[B]$ is the expected number of back-off slots that a packet waits before transmission; and $E[T]$ is the average slot duration and it can be calculated by averaging the duration of each type of event (e.g., transmission, collision) with its probability. The reader is encouraged to read a more detailed derivation of all these parameters in our previous works $[15,16,40]$. Finally, $\lambda$ and $L_{\mathrm{PDU}}$ represent the number of packets transmitted per second and their payload length, respectively (e.g., 50 packets/second and 160 Bytes for G.711 codec).

Finally, (2) and (3) can be completed as

$$
\begin{gathered}
P_{\mathrm{pl}}=1-\left(\left(1-L_{\mathrm{WiFi}}\left(\vec{d}_{i}\right)\right)\left(1-L_{\mathrm{WiFi}}\left(\vec{a}_{j}\right)\right)\left(1-L_{5 \mathrm{G}-\mathrm{SLA}}\right)\right), \\
d=20 \mathrm{~ms}+D_{\mathrm{WiFi}}\left(\vec{d}_{i}\right)+D_{\mathrm{WiFi}}\left(\vec{a}_{j}\right)+d_{5 \mathrm{G}-\mathrm{SLA}},
\end{gathered}
$$

where $20 \mathrm{~ms}$ accounts for the packetization interval for the G.711 codec. Once computed, $P_{\mathrm{pl}}$ and $d$ can be further substituted in (1) to obtain $R$.

5.2.3. Computational Complexity. In terms of computational complexity, the most demanding procedure in our heuristic search is the fitness evaluation (Check function). If we assume that $D$ is upper bounded by $A$ and that the maximum number of calls to the Check function is upper bounded in our heuristics, the computational complexity 

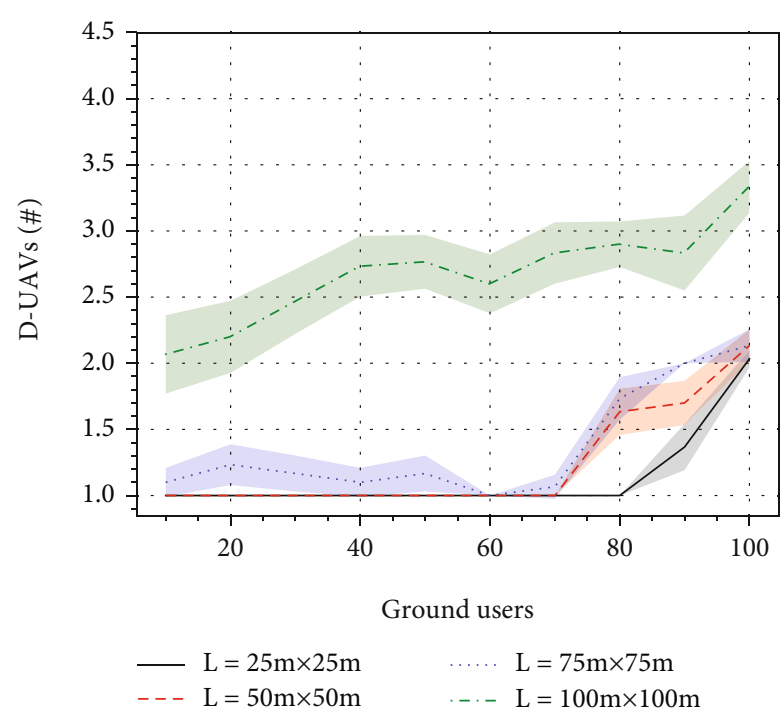

(a) Number of deployed D-UAVs

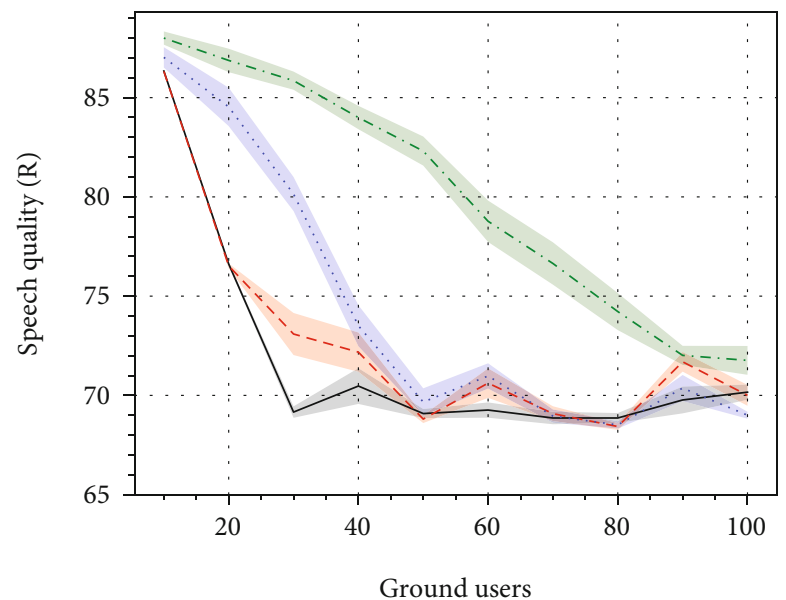

$$
\begin{array}{ll}
-\mathrm{L}=25 \mathrm{~m} \times 25 \mathrm{~m} & \ldots . . \mathrm{L}=75 \mathrm{~m} \times 75 \mathrm{~m} \\
--\mathrm{L}=50 \mathrm{~m} \times 50 \mathrm{~m} & \ldots-\mathrm{L}=100 \mathrm{~m} \times 100 \mathrm{~m}
\end{array}
$$

(c) Speech quality

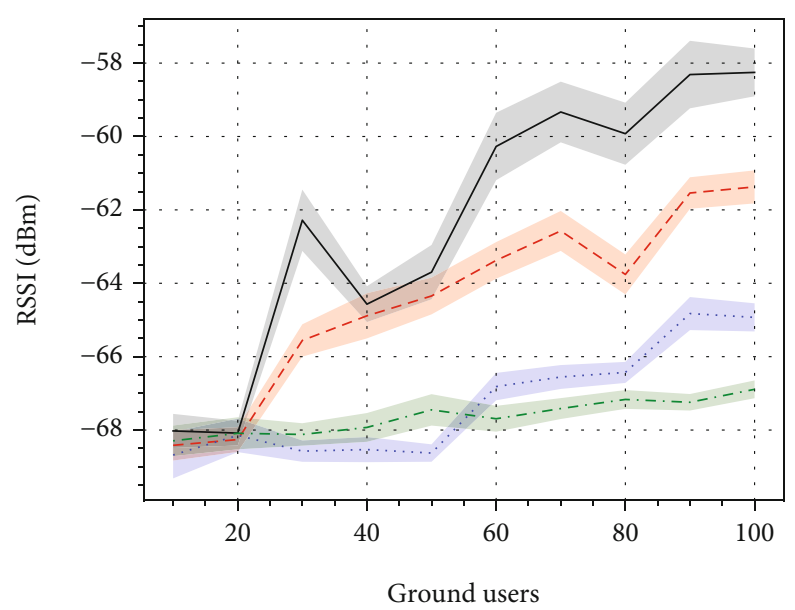

$$
\begin{aligned}
& -\mathrm{L}=25 \mathrm{~m} \times 25 \mathrm{~m} \quad \ldots . . \mathrm{L}=75 \mathrm{~m} \times 75 \mathrm{~m} \\
& \ldots \mathrm{L}=50 \mathrm{~m} \times 50 \mathrm{~m} \quad \ldots-\mathrm{L}=100 \mathrm{~m} \times 100 \mathrm{~m}
\end{aligned}
$$

(e) Average RSSI at ground users

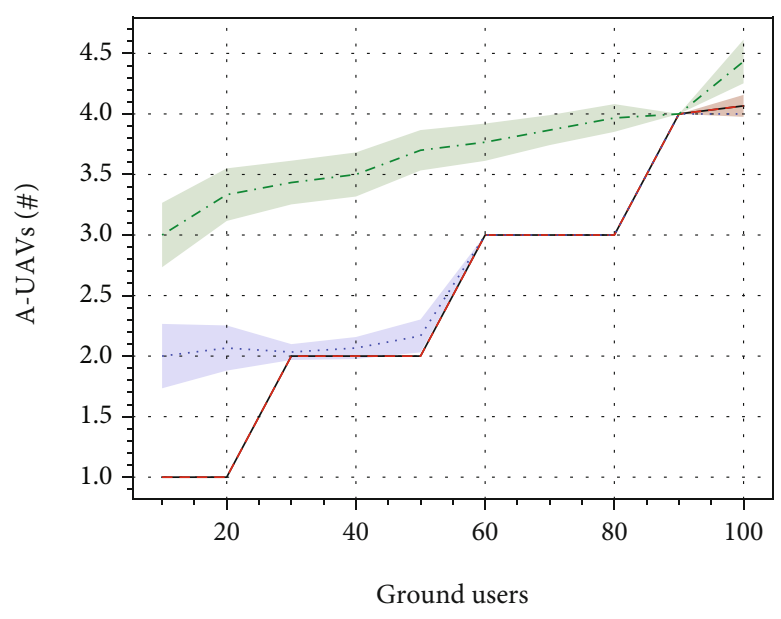

$\begin{aligned}-\mathrm{L}=25 \mathrm{~m} \times 25 \mathrm{~m} & \ldots \ldots \mathrm{L}=75 \mathrm{~m} \times 75 \mathrm{~m} \\ --\mathrm{L}=50 \mathrm{~m} \times 50 \mathrm{~m} & \ldots-. \quad \mathrm{L}=100 \mathrm{~m} \times 100 \mathrm{~m}\end{aligned}$

(b) Number of deployed A-UAVs

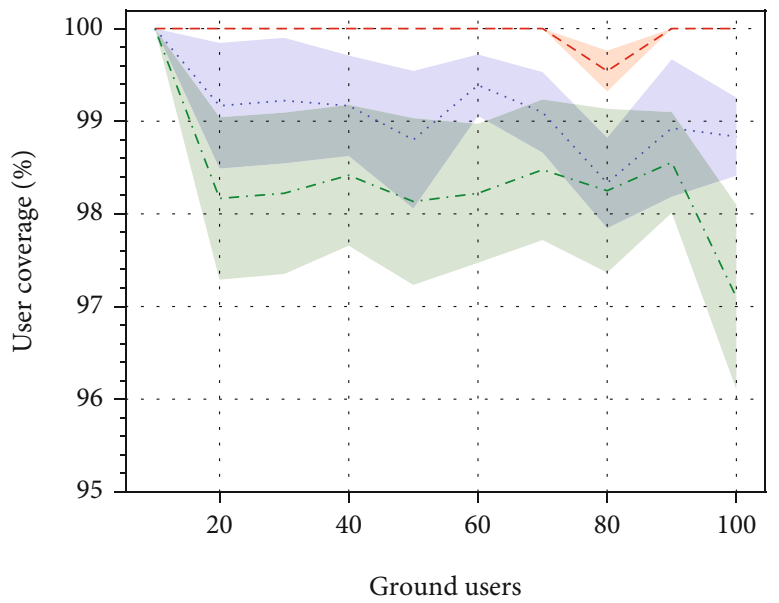

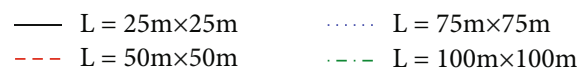

(d) Percentage of covered users

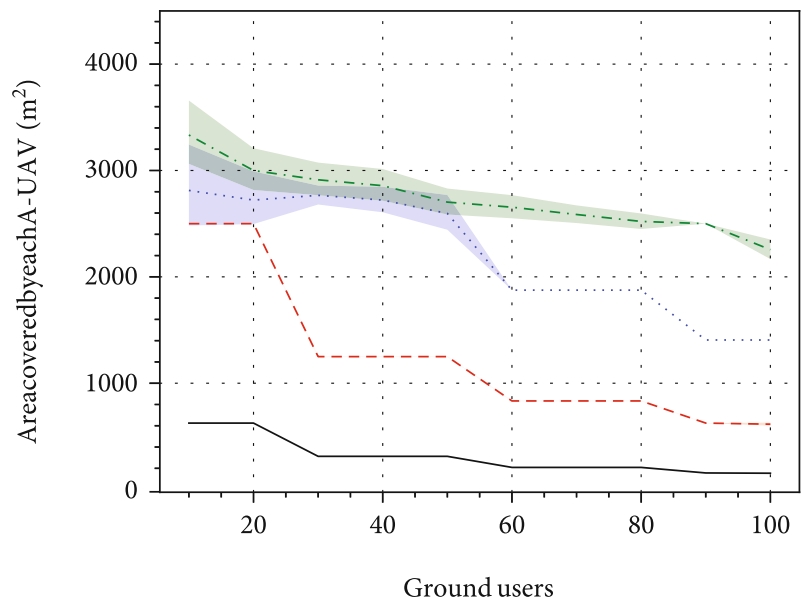

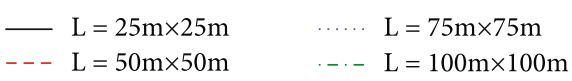

(f) Average area covered by A-UAVs

Figure 4: Numerical results. 
of the SolveOptim_PSO function can be expressed as $\mathcal{O}$ $(A \cdot U)$; hence, the computational complexity of Algorithm 5.1 can be expressed as $\mathcal{O}\left(D \cdot A^{2} \cdot U\right)$.

\section{Results}

We have used our PSO-based search algorithm to find optimal solutions in different scenarios. This section begins with a $100 \mathrm{~m} \times 100 \mathrm{~m}$ scenario and 60 ground users for illustrative purposes. Then, we explore other scenarios changing the terrain size (from $25 \mathrm{~m} \times 25 \mathrm{~m}$ to $100 \mathrm{~m} \times 100 \mathrm{~m}$ ) and the number of users (from 10 to 100).

6.1. Example Solution. This section provides a first example solution in a $100 \mathrm{~m} \times 100 \mathrm{~m}$ terrain with 60 ground users randomly placed. The rest of the parameters used are listed in Table 3. Such settings are common to all experiments in this section unless otherwise specified.

The PSO-based search algorithm in Algorithm 2 has been implemented in Matlab 2020a, providing the results shown in Figure 3. In this scenario, four A-UAVs and two D-UAVs are required to cover 58 of the 60 ground users while guaranteeing a minimal speech quality of 65 (R factor).

In Figure 3, ground users are represented with circles filled with color (if covered) or not (if uncovered). Each ground user is associated with an A-UAV of the same color whose location has been derived from the algorithm. Finally, each A-UAV is associated with a D-UAV in a similar manner. As such, colors represent the final association between different layers. According to Figure 3, two 5G links would suffice to serve the 58 ground users under coverage.

6.2. Numerical Analysis. In this section, we examine the solutions found in several scenarios. In particular, the number of ground users has been increased from 10 to 100 (in steps of 10) in four square terrain sizes $(25 \mathrm{~m} \times 25 \mathrm{~m}, 50 \mathrm{~m}$ $\times 50 \mathrm{~m}, 75 \mathrm{~m} \times 75 \mathrm{~m}$, and $100 \mathrm{~m} \times 100 \mathrm{~m}$ ). Each experiment (i.e., scenario) has been repeated 30 times to obtain a $95 \%$ confidence interval by randomly placing ground users uniformly along the terrain. Finally, Figure 4 illustrates the average value of the results obtained with the corresponding confidence interval.

Figures 4(a) and 4(b) show the optimal number of DUAVs and A-UAVs, respectively, for different numbers of users $(U)$ and terrain sizes. At first sight, it can be observed that the overall number of UAVs tends to grow when either the terrain size or the number of users increases.

If we take a closer look into the $50 \mathrm{~m} \times 50 \mathrm{~m}$ series, the number of A-UAVs experiences a steep growth after 20 users. This effect is due to network congestion, so more UAVs are required to satisfy the speech quality constraints. This behavior is reaffirmed in Figure 4(c), which represents the speech quality, and shows that speech quality degrades when more users are added to the system. Indeed, after 70 users, more D-UAVs are required to aggregate the traffic from the access network so that QoS is not negatively affected. In our results, it is likely that each D-UAV associates with up to three A-UAV hence reducing the number of $5 \mathrm{G}$ links required to provide the service. Although this

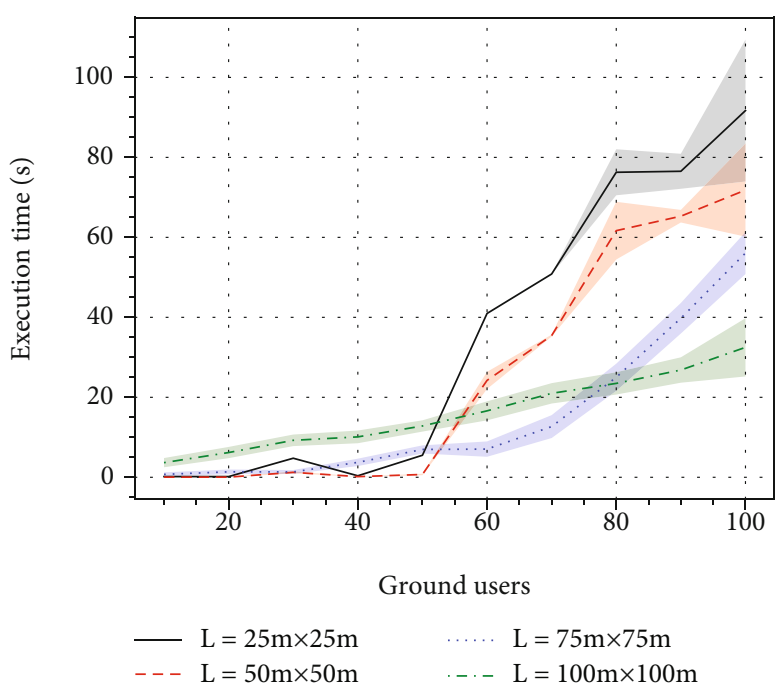

FIgURe 5: Performance analysis.

behavior is similar various terrain sizes, the final number of UAVs changes with user sparsity $\left(\mathrm{m}^{2} / \mathrm{U}\right)$; if the terrain is increased with the same number of ground users, UAVs have to cover a larger area to satisfy coverage constraints $\left(C / U \geq C_{\min }\right)$; hence, the number of drones is driven by the signal coverage constraint instead of the speech quality constraint.

Figure 4(d) shows the percentage of users covered in each scenario. Our results suggest that larger areas tend to provide lower ratios of user coverage due to a greater distance between users and A-UAVs. This, in turn, penalizes the path loss. Figure 4(e) represents the average received signal strength (RSSI) for each series of experiments. As illustrated, the RSSI increases with the number of ground users. This is attributable to the fact that requiring more A-UAVs to meet the speech quality requirements (e.g., reduce congestion) also produces UAVs located closer to users, hence increasing RSSI values. As a consequence, the effective area covered by each A-UAV is reduced (see Figure 4(f)).

6.3. Computational Performance. The previous series of experiments have also been analyzed in light of computational performance. To this end, we measured the average time spent during the execution of the algorithm. Figure 5 illustrates the execution time in seconds for the same series of experiments in an Intel Core $^{\mathrm{TM}}$ i5-4460 processor with $8 \mathrm{~GB}$ of RAM.

The results shown in Figure 5 suggest that the execution time increases with user density $\left(\mathrm{U} / \mathrm{m}^{2}\right)$ for a given terrain size, so more dense scenarios require more CPU time. However, the execution time is almost linear when solving low user-density scenarios (e.g., $100 \mathrm{~m} \times 100 \mathrm{~m}$ ). In all cases, the results were obtained in less than 100 seconds.

\section{Conclusion and Further Steps}

This paper has dealt with the creation of a drone-assisted VoWiFi communication service, which could be of use in 
cases such as emergency situations or covering live events that lack better alternative infrastructure. We have defined a new optimization problem for the deployment of drones to create a two-layer network (access and distribution) connected to 5G. Our problem is aimed at finding the minimum number of drones (and their type and location) required to deploy the service constrained to a minimum user coverage and speech quality.

Using a PSO-based search algorithm, we have calculated the optimal solution for terrains up to $100 \mathrm{~m} \times 100 \mathrm{~m}$ and 100 users with moderate computational resources. The overall number of UAVs tends to increase with the terrain size (i.e., with users' sparsity) or with the number of users. The next step planned is to explore new applications in this field: (a) to implement a model-based Call Admission Control (CAC) algorithm in UAVs, so new calls can be rejected if the network is saturated; and (b) to explore new applications of the 3-D UAV optimal placement, such as qualityguaranteed videocalls or IoT sensing through drones, that might require a different network architecture.

\section{Data Availability}

No data were used to support this study.

\section{Conflicts of Interest}

The authors declare that there is no conflict of interest regarding the publication of this article.

\section{References}

[1] M. Hassanalian and A. Abdelkefi, "Classifications, applications, and design challenges of drones: a review," Progress in Aerospace Sciences, vol. 91, pp. 99-131, 2017.

[2] R. Clarke, "Understanding the drone epidemic," Computer Law and Security Review, vol. 30, no. 3, pp. 230-246, 2014.

[3] F. Al-Turjman, M. Abujubbeh, A. Malekloo, and L. Mostarda, "UAVs assessment in software-defined IoT networks: an overview," Computer Communications, vol. 150, pp. 519-536, 2020.

[4] R. Masroor, M. Naeem, and W. Ejaz, "Resource management in UAV-assisted wireless networks: an optimization perspective," Ad Hoc Networks, vol. 121, article 102596, 2021.

[5] R. Shahzadi, M. Ali, H. Z. Khan, and M. Naeem, "UAV assisted 5G and beyond wireless networks: a survey," Journal of Network and Computer Applications, vol. 189, article 103114, 2021.

[6] M. Mozaffari, W. Saad, M. Bennis, Y.-H. Nam, and M. Debbah, "A tutorial on uavs for wireless networks: applications, challenges, and open problems," IEEE Communication Surveys and Tutorials, vol. 21, no. 3, pp. 2334-2360, 2019.

[7] I. Dalmasso, I. Galletti, R. Giuliano, and F. Mazzenga, "Wimax networks for emergency management based on UAVs," in 2012 IEEE First AESS European Conference on Satellite Telecommunications (ESTEL), pp. 1-6, Rome, Italy, 2012.

[8] S. Rohde, M. Putzke, and C. Wietfeld, "Ad hoc self-healing of OFDMA networks using UAV-based relays," Ad Hoc Networks, vol. 11, no. 7, pp. 1893-1906, 2013.
[9] S. K. Khan, U. Naseem, H. Siraj, I. Razzak, and M. Imran, "The role of unmanned aerial vehicles and mmWave in $5 \mathrm{~g}$ : recent advances and challenges," Transactions on Emerging Telecommunications Technologies, vol. 32, no. 7, article e4241, 2021.

[10] S. Morgenthaler, T. Braun, Z. Zhao, T. Staub, and M. Anwander, "Uavnet: a mobile wireless mesh network using unmanned aerial vehicles," in 2012 IEEE Globecom Workshops, pp. 1603-1608, Anaheim, CA, USA, 2012.

[11] S. Aust, R. V. Prasad, and I. G. Niemegeers, "Outdoor long-range wlans: a lesson for IEEE 802.11ah," IEEE Communication Surveys and Tutorials, vol. 17, no. 3, pp. 17611775, 2015.

[12] M. S. Alvissalim, B. Zaman, Z. A. Hafizh et al., "Swarm quadrotor robots for telecommunication network coverage area expansion in disaster area," in 2012 Proceedings of SICE Annual Conference (SICE), pp. 2256-2261, Akita, Japan, 2012.

[13] M. Erdelj, M. Król, and E. Natalizio, "Wireless sensor networks and multi-UAV systems for natural disaster management," Computer Networks, vol. 124, pp. 72-86, 2017.

[14] H. Shakhatreh, A. H. Sawalmeh, A. Al-Fuqaha et al., "Unmanned aerial vehicles (UAVS): a survey on civil applications and key research challenges," IEEE Access, vol. 7, pp. 48572-48634, 2019.

[15] V. Mayor, R. Estepa, A. Estepa, and G. Madinabeitia, “Deploying a reliable UAV-aided communication service in disaster areas," Wireless Communications and Mobile Computing, vol. 2019, Article ID 7521513, 20 pages, 2019.

[16] "Energy-efficient UAVs deployment for QoS-guaranteed VoWiFi service," Sensors, vol. 20, no. 16, p. 4455, 2020.

[17] Y. Li, G. Feng, M. Ghasemiahmadi, and L. Cai, "Power allocation and 3-d placement for floating relay supporting indoor communications," IEEE Transactions on Mobile Computing, vol. 18, no. 3, pp. 618-631, 2019.

[18] C. Pan, C. Yin, J. Yu, and N. Kiran, "3d UAV placement and resource allocation in software defined cellular networks," in 2018 IEEE/CIC International Conference on Communications in China (ICCC Workshops), pp. 136-141, Beijing, China, 2018.

[19] C. Pan, J. Yi, C. Yin, J. Yu, and X. Li, "Joint 3d UAV placement and resource allocation in software-defined cellular networks with wireless backhaul," IEEE Access, vol. 7, pp. 104279104293, 2019.

[20] S. Yin, L. Li, and F. R. Yu, "Resource allocation and basestation placement in downlink cellular networks assisted by multiple wireless powered UAVs," IEEE Transactions on Vehicular Technology, vol. 69, no. 2, pp. 2171-2184, 2019.

[21] Y. Sun, T. Wang, and S. Wang, "Location optimization and user association for unmanned aerial vehicles assisted mobile networks," IEEE Transactions on Vehicular Technology, vol. 68, no. 10, pp. 10056-10065, 2019.

[22] X. Liu, J. Wang, N. Zhao et al., "Placement and power allocation for NOMA-UAV networks," IEEE Wireless Communications Letters, vol. 8, no. 3, pp. 965-968, 2019.

[23] D. Hu, Q. Zhang, Q. Li, and J. Qin, “Joint position, decoding order, and power allocation optimization in UAV-based NOMA downlink communications," IEEE Systems Journal, vol. 14, no. 2, pp. 2949-2960, 2019.

[24] H. Zhao, H. Wang, W. Wu, and J. Wei, "Deployment algorithms for UAV airborne networks toward on-demand coverage," IEEE Journal on Selected Areas in Communications, vol. 36, no. 9, pp. 2015-2031, 2018. 
[25] H. Wang, H. Zhao, W. Wu, J. Xiong, D. Ma, and J. Wei, "Deployment algorithms of flying base stations: $5 \mathrm{~g}$ and beyond with UAVS," IEEE Internet of Things Journal, vol. 6, no. 6, pp. 10009-10027, 2019.

[26] J. Chakareski, S. Naqvi, N. Mastronarde, J. Xu, F. Afghah, and A. Razi, "An energy efficient framework for UAV-assisted millimeter wave $5 \mathrm{~g}$ heterogeneous cellular networks," IEEE Transactions on Green Communications and Networking, vol. 3, no. 1, pp. 37-44, 2019.

[27] S. Zeng, H. Zhang, and L. Song, "Trajectory optimization and resource allocation for multi-user OFDMA UAV relay networks," in 2019 IEEE Global Communications Conference (GLOBECOM), pp. 1-6, Waikoloa, HI, USA, 2019.

[28] H. Mei, K. Yang, Q. Liu, and K. Wang, "Joint trajectoryresource optimization in UAV-enabled edge-cloud system with virtualized mobile clone," IEEE Internet of Things Journal, vol. 7, no. 7, pp. 5906-5921, 2019.

[29] R. K. Patra and P. Muthuchidambaranathan, "Optimisation of spectrum and energy efficiency in UAV-enabled mobile relaying using bisection and PSO method," in 2018 3rd International Conference for Convergence in Technology (I2CT), pp. 1-7, Pune, India, 2018.

[30] J. Zhang, Y. Zeng, and R. Zhang, "Spectrum and energy efficiency maximization in UAV-enabled mobile relaying," in 2017 IEEE International Conference on Communications (ICC), pp. 1-6, Paris, France, 2017.

[31] Q. Hu, Y. Cai, A. Liu, and G. Yu, "Joint resource allocation and trajectory optimization for UAV-aided relay networks," in 2019 IEEE Global Communications Conference (GLOBECOM), pp. 1-6, Waikoloa, HI, USA, 2019.

[32] M. Samir, M. Chraiti, C. Assi, and A. Ghrayeb, "Joint optimization of UAV trajectory and radio resource allocation for drive-thru vehicular networks," in 2019 IEEE Wireless Communications and Networking Conference (WCNC), pp. 1-6, Marrakesh, Morocco, 2019.

[33] Q. Chen, "Joint trajectory and resource optimization for UAVenabled relaying systems," IEEE Access, vol. 8, pp. 2410824119, 2020.

[34] X. Jiang, Z. Wu, Z. Yin, and Z. Yang, "Power and trajectory optimization for UAV-enabled amplify-and-forward relay networks," IEEE Access, vol. 6, pp. 48688-48696, 2018.

[35] G. Zhang, H. Yan, Y. Zeng, M. Cui, and Y. Liu, “Trajectory optimization and power allocation for multi-hop UAV relaying communications," IEEE Access, vol. 6, pp. 48566-48576, 2018.

[36] S. Zeng, H. Zhang, K. Bian, and L. Song, "UAV relaying: power allocation and trajectory optimization using decodeand-forward protocol," in 2018 IEEE International Conference on Communications Workshops (ICC Workshops), pp. 1-6, Kansas City, MO, USA, 2018.

[37] S. Karapantazis and F.-N. Pavlidou, "VoIP: a comprehensive survey on a promising technology," Computer Networks, vol. 53, no. 12, pp. 2050-2090, 2009.

[38] ITU-T, Recommendation G and Recommendation, G, “107: The E-model, a computational model for use in transmission planning," Recommendation ITU-T, 2005.

[39] H. Assem, D. Malone, J. Dunne, and P. O’Sullivan, “Monitoring VOIP call quality using improved simplified e-model,", in 2013 International Conference on Computing, Networking and Communications (ICNC), pp. 927-931, San Diego, CA, USA, 2013.
[40] V. Mayor, R. Estepa, A. Estepa, and G. Madinabeitia, "Unified call admission control in corporate domains," Computer Communications, vol. 150, pp. 589-602, 2020.

[41] E. Hossain and M. Hasan, "5G cellular: key enabling technologies and research challenges," IEEE Instrumentation and Measurement Magazine, vol. 18, no. 3, pp. 11-21, 2015.

[42] Sangho Shin and H. Schulzrinne, "Measurement and analysis of the VOIP capacity in IEEE 802.11 WLAN," IEEE Transactions on Mobile Computing, vol. 8, no. 9, pp. 1265-1279, 2009.

[43] J. Sánchez-García, D. G. Reina, and S. L. Toral, “A distributed PSO-based exploration algorithm for a UAV network assisting a disaster scenario," Future Generation Computer Systems, vol. 90, pp. 129-148, 2019.

[44] A. Al-Hourani, S. Kandeepan, and A. Jamalipour, "Modeling air-toground path loss for low altitude platforms in urban environments," in 2014 IEEE Global Communications Conference, pp. 2898-2904, Austin, TX, USA, 2014.

[45] A. Al-Hourani, S. Kandeepan, and S. Lardner, "Optimal lap altitude for maximum coverage," IEEE Wireless Communications Letters, vol. 3, no. 6, pp. 569-572, 2014.

[46] M. Mozaffari, W. Saad, M. Bennis, and M. Debbah, "Drone small cells in the clouds: design, deployment and performance analysis," in 2015 IEEE Global Communications Conference (GLOBECOM), pp. 1-6, San Diego, CA, USA, 2015.

[47] Q. Feng, J. McGeehan, E. K. Tameh, and A. R. Nix, "Path loss models for air-to-ground radio channels in urban environments," in 2006 IEEE 63rd Vehicular Technology Conference, pp. 2901-2905, Melbourne, VIC, Australia, 2006.

[48] Y. Zheng, Y. Wang, and F. Meng, "Modeling and simulation of pathloss and fading for air-ground link of haps within a network simulator," in 2013 International Conference on CyberEnabled Distributed Computing and Knowledge Discovery, pp. 421-426, Beijing, China, 2013.

[49] G. Bianchi, "Performance analysis of the IEEE 802.11 distributed coordination function," IEEE Journal on Selected Areas in Communications, vol. 18, no. 3, pp. 535-547, 2000. 